\title{
Morphological Changes in Nasal Mucosa in Patients with Sarcoidosis.
}

\author{
Pavlos Pavlidis ${ }^{1}$, Evangelia Fouka ${ }^{2}$, Georgios Katsilis ${ }^{1}$, Haralampos Gouveris ${ }^{3}$, and \\ Despoina Papakosta ${ }^{2}$ \\ ${ }^{1}$ General Hospital of Thessaloniki G Papanikolaou \\ ${ }^{2}$ General Hospital of Thessaloniki G Papanikolaou Pulmonology Department NHS \\ ${ }^{3}$ Johannes Gutenberg University Hospital Mainz
}

March 29, 2021

\begin{abstract}
Objectives: The purpose of this study was the evaluation of any alterations in the microvascular network of the nasal mucosa in patients with pulmonary sarcoidosis and the investigation of potential correlations with olfactory acuity and serum levels of angiotensin-converting enzyme (sACE). Design: Patients' nasal mucosa was examined with contact endoscopy (CE). A novel classification scheme for the microvascular pattern at the anterior septal mucosa (Little's area) was introduced and implemented. Olfaction was tested using sniffin'sticks. Fifteen healthy subjects served as controls. Participants: 15 patients with pulmonary sarcoidosis and sinonasal symptoms. Main outcome measures: Microvascular pattern at the anterior septal mucosa (Little's area). Olfaction tested using sniffin'sticks. Setting: Tertiary referral medical centre. Results: The nasal microvascular network was disrupted under CE in most (14/15) patients, while in one patient no microvascular net could be detected. Moreover, hyposmia was documented in four patients and complete anosmia in one patient. In healthy subjects, a very strong correlation between vascular pattern of the mucosa and olfactory test results was found $(r=0.93)$. Conclusions: Contact endoscopy findings show promise and should be further tested, to evaluate their validity as a surrogate marker of mucosal nasal inflammation in sarcoidosis patients with sinonasal symptoms. Vascular patterns of nasal mucosa and olfaction seem to be strong correlated.
\end{abstract}

\section{Hosted file}

nasal sarcoidosis10032021.pdf available at https://authorea.com/users/313079/articles/515806morphological-changes-in-nasal-mucosa-in-patients-with-sarcoidosis

\section{Hosted file}

Table 1.pdf available at https://authorea.com/users/313079/articles/515806-morphologicalchanges-in-nasal-mucosa-in-patients-with-sarcoidosis

\section{Hosted file}

Table 2.pdf available at https://authorea.com/users/313079/articles/515806-morphologicalchanges-in-nasal-mucosa-in-patients-with-sarcoidosis 
$\rightarrow \quad 4$
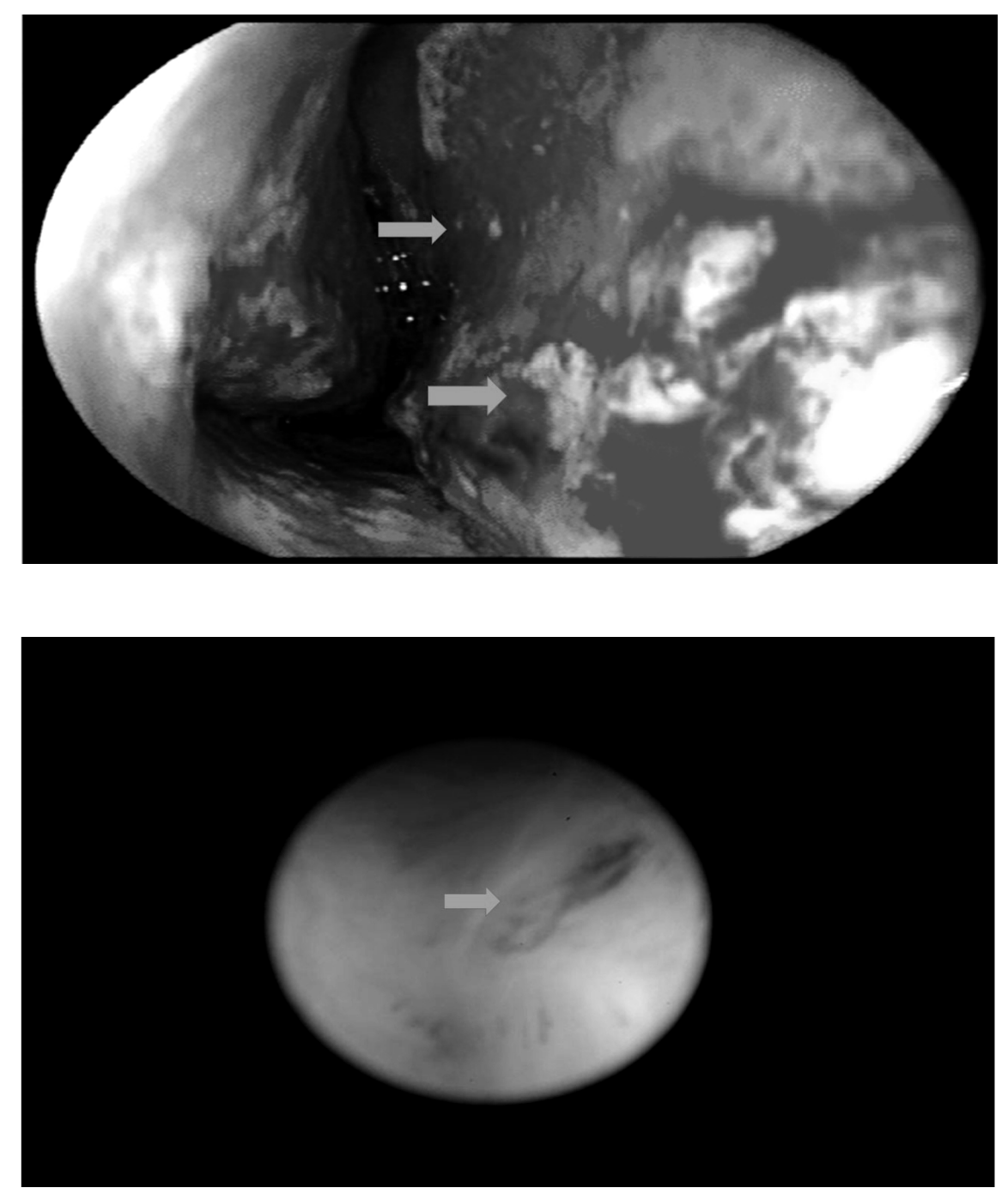